\title{
Koffka's Ring Effect Depends on Thickness, Not Continuity
}

\author{
Abigail E. Huang ${ }^{1 *}$, Alice J. Hon ${ }^{1 *}$, Eric Lewin Altschuler ${ }^{1,2}$ \\ ${ }^{1}$ School of Medicine, ${ }^{2}$ Department of Physical Medicine and Rehabilitation, University of \\ Medicine \& Dentistry of New Jersey, Newark, New Jersey, 07103.
}

*These authors contributed equally to the work.

Correspondence to Eric Lewin Altschuler, $\mathrm{MD}, \mathrm{PhD}$

Department of Physical Medicine and Rehabilitation

University of Medicine \& Dentistry of New Jersey

University Hospital, B-403

150 Bergen Street

Newark, NJ 07103

Phone: (973) 972-5439

Fax: (973) 972-5725

email: eric.altschuler@umdnj.edu 
More than 70 years ago Gestalt psychologist Kurt Koffka described a fascinating effect $^{1,2}$ : When a contiguous grey ring is placed on a background half of one shade of grey (different from the ring) and half of another shade of grey, the ring appears to be a homogenous. However, if the ring is slightly divided, now the two halves of the ring appear different shades of grey with the half of the ring on the darker background appearing lighter than the half of the ring on the darker background. The Gestalt principle of continuity in visual perception is invoked to explain this effect. Here we show that in fact when the ring is made thinner it appears heterogeneous even when contiguous. Furthermore, when viewing a thick ring after first viewing a thin ring, the thick ring now too appears heterogenous! These effects are also demonstrated with a colored background and backgrounds with more than two regions. We show that standard simultaneous brightness and color contrast weaken with larger test patches. Thus, Koffka's ring effect is due to vanishingly weak simultaneous contrast for a sufficiently thick ring, not continuity.

The standard version of the effect is given in Fig. 1. In the left panel notice that the ring appears to be a homogeneous color, while in the right panel even the slightest separation between the halves of the rings causes the left half of the ring to appear lighter than the right half of the ring, even though the two greys are physically identical. The half of the ring on the darker background appears lighter than the half of the ring on the lighter background consistent with standard contrast effects. (We have found that some people can see some heterogeneity of the solid ring, but significantly weaker than for the divided ring and also with a much less sharp boundary.) Our main result is given in Fig. 2. The 
bottom ring is a standard setup of a contiguous grey ring on a half light and half dark grey background, but here the ring is thinner than the typical ring used to illustrate Koffka's effect. In this case, interestingly, the contiguous ring appears to be colored two shades of grey - the right half on the lighter grey background being darker than the left half on the darker grey background. The middle ring of Fig. 2, an intermediate thickness contiguous ring, again appears to be of two colors like the bottom ring. Finally, remarkably, upon now looking at the top ring, one of the thickness used to demonstrate Koffka's effect in Fig. 1, now too appears to be split colored. This hysteresis of the perception of the ring as heterogeneous is also seen in Fig. 3 moving from panels right to left and bottom to top. Viewing panels from left to right and top to bottom lets one find the critical annulus thickness where the ring crosses over from appearing homogenous to heterogeneous.

A colored version of our new effects is given in Fig. 4. In the top panel of Fig. 4a the grey ring appears to be homogenous grey, while in the bottom panel the right side appears a lighter grey while the left side appears to be cyan (= green + blue). However, the bottom ring of Fig. 4b, the thin contiguous grey ring, again appears to be heterogeneous with the right side appearing a light grey, and the left side cyan. The middle ring of intermediate thickness is likewise heterogeneous, and again upon next viewing the top ring (of thickness of that in Fig. 4a) there is a hysteresis effect such that the thick contiguous ring appears heterogeneous too. Figs. 4c and 4d illustrate Koffka's effect and our new effects for a two colored background, and Figs. 5a and 5b for a background divided into quadrants. 
Why does the standard sized contiguous ring appear a homogenous color? Our thinking was that the ring typically used to demonstrate Koffka's effect was sufficiently thick that simultaneous brightness would be too weak to be appreciated. Indeed, as shown in Fig. 6 that typical simultaneous brightness and color contrast displays that weaken with increasing width of the test patch. Why does a thick ring that is slightly divided appear heterogeneous? The sharp boundary edge must be strongly respected by the neural mechanisms of simultaneous contrast. The explanation for the hysteresis/persistence effect we have found of viewing a thin ring as being heterogeneous and then the thick ring appearing heterogeneous as well is not yet completely clear, but we have found some properties of the effect. First, the appearance of heterogeneity of the thick ring fades significantly after some seconds. Also, shown in Fig. 7 the hysteresis effect is not just an afterimage effect. Finally, the hysteresis is occurring at a level higher than the retina, as if one looks at the thin and intermediate thickness rings in Fig. 2 with one eye, and then looks at the thick ring (with the thin and intermediate thickness rings covered up) with the other eye the thick ring appears heterogeneous.

The Gestalt psychologists ${ }^{1,3}$ were brilliant in describing many effects and illusions that are simple in their display but touch on effects of the visual and other perceptual and cognitive systems which are most fundamental and thought provoking. Approaching a century on many remain partially or wholly unexplained. One question which arises about Gestalt effects and Gestalt explanatory principles is whether they are fundamental rules or laws, or "just" absolutely ingenious kluges or macroscopic phenomenologic rules of thumb with deeper underlying microscopic rules (or like Bohr's complementarity 
principle of quantum mechanics a bridge between a very non-intuitive microscopic theory and seemingly simpler macroscopic phenomena). The brain does not give up her secrets readily, but at least in the case of Koffka's ring effect we see that continuity of the ring does not alone produce a homogenous ring but a sufficiently thick ring such that simultaneous contrast is not perceptible. The hysteresis effect on the thick ring of first viewing the thin apparently heterogeneous ring awaits full understanding of its microscopic basis. 


\section{References}

1.Koffka, K. Principles of Gestalt Psychology (Lund Humphries, London, 1935).

2.Adelson, E. H. in The New Cognitive Neurosciences $2^{\text {nd }}$ Edition (ed Gazzaniga, M.) 339-351 (MIT Press, Cambridge, MA, 2000).

3.Köhler, W. Gestalt Psychology (G. Bell \& Sons, Ltd., London, 1930). 

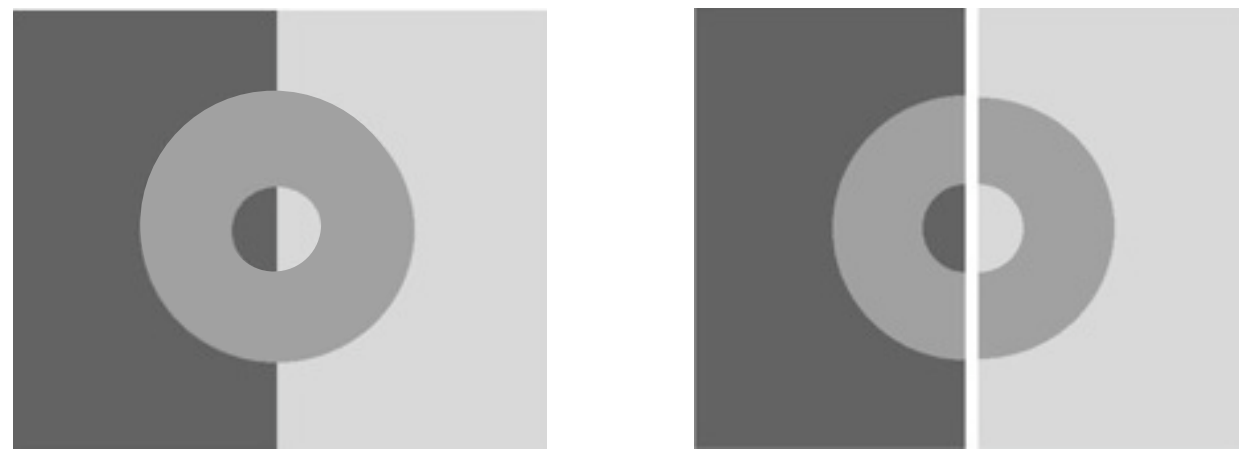

Figure 1 Koffka's ring effect. Notice that on the left the contiguous grey ring appears to be a homogeneous color, while on the right by dividing the ring slightly the two halves appear to be different shades of grey. 


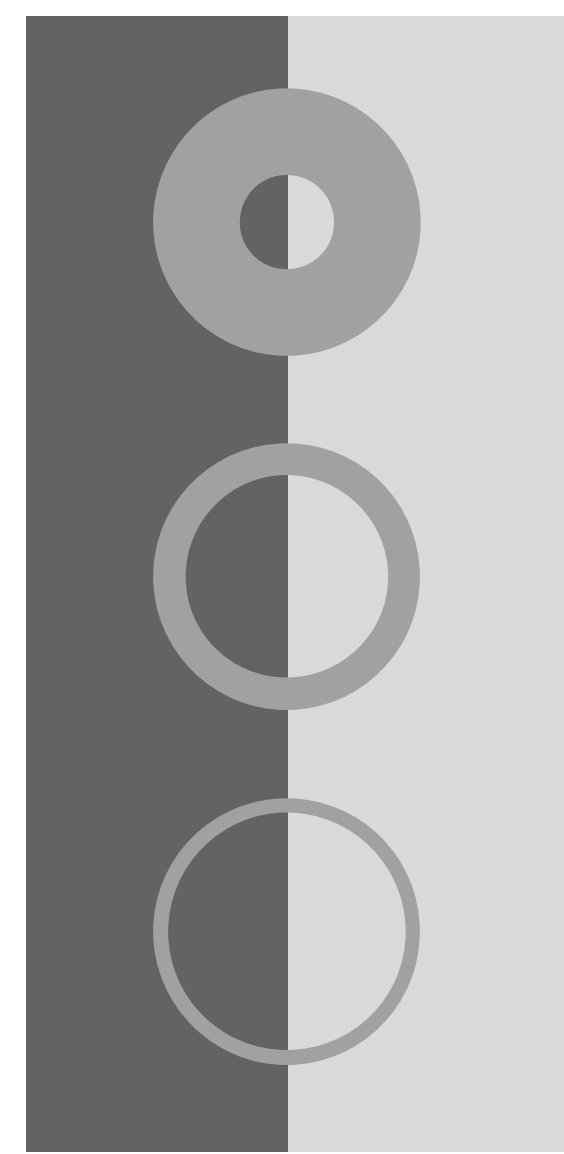

Figure 2 Koffka's ring effect depends on ring thickness not continuity. Look first at the bottom, then the middle, then the top ring. The bottom ring, despite being contiguous appears to be heterogeneous with each half of the ring being a different shade. The middle ring looks likewise. Remarkably, the top ring, despite being as thick as the one in Fig. 1 now too looks to be heterogeneous. 
Figure 3 Critical ring

thickness. The ring thickness at which the ring clearly appears heterogeneous varies somewhat from individual to individual and with viewing conditions, but we see the ring as heterogeneous by the second from the top ring on the right. Start from the left (right) panel and from top to bottom (bottom to top) to avoid (experience) the hysteresis effect. The third ring from the top on the left is the thickness of the ring in Fig. 1.
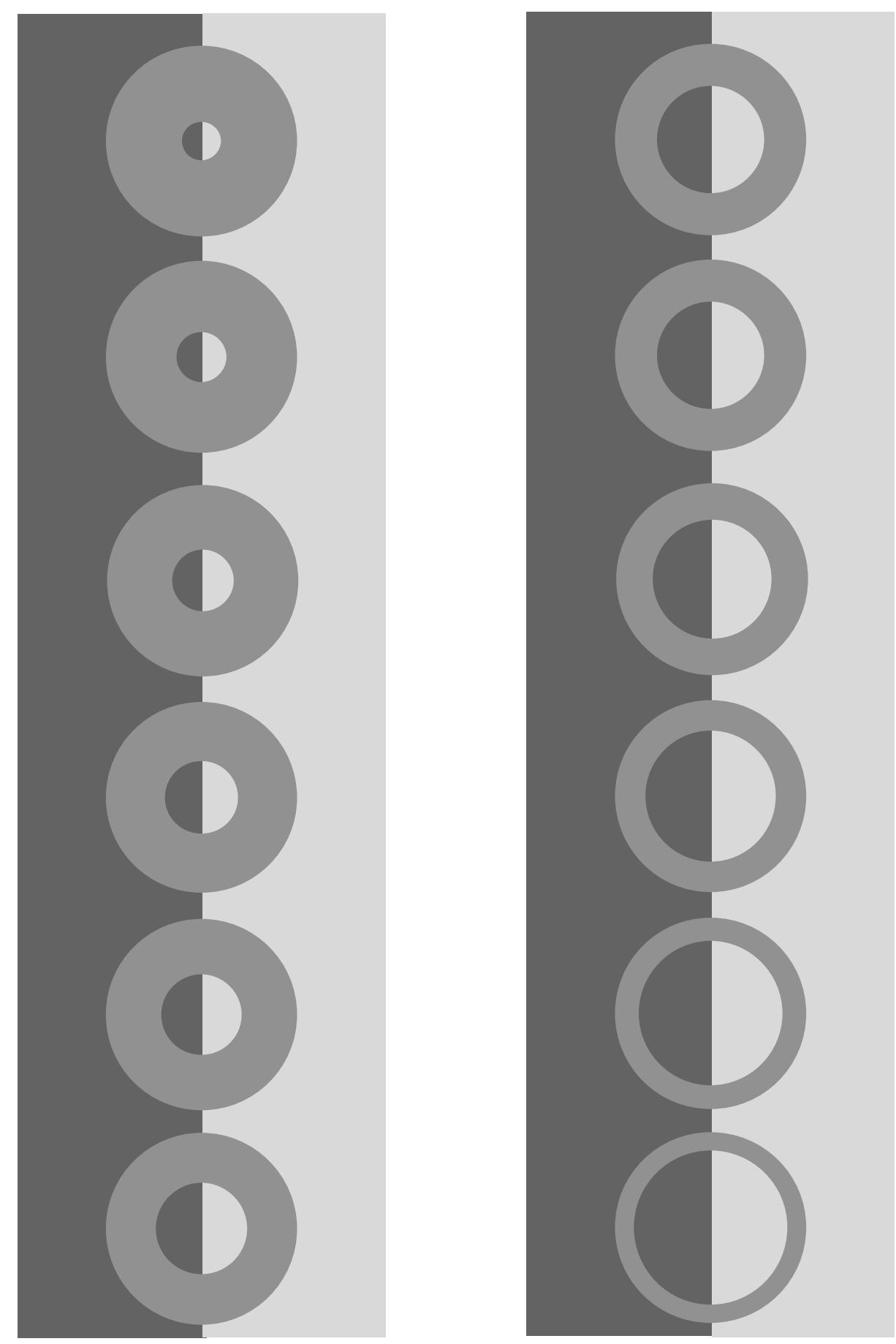

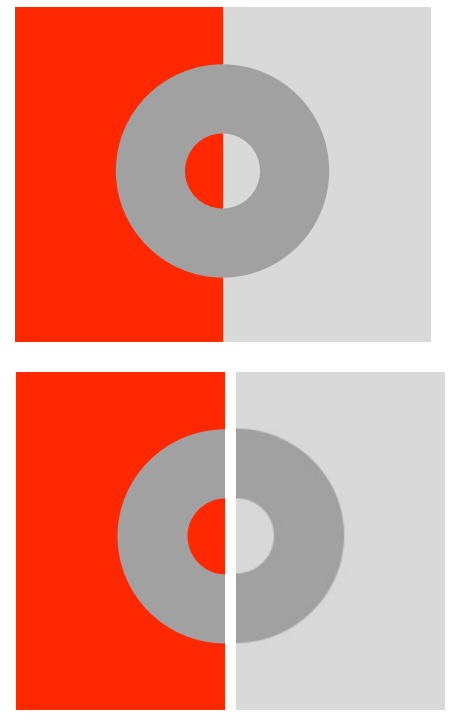

$\mathbf{a}$

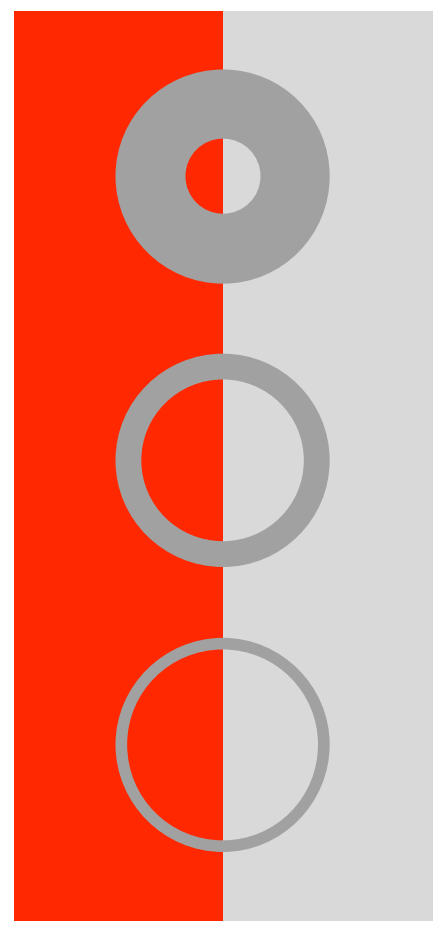

b

Figure 4 Colored version of the heterogeneous contiguous ring. a, The standard set-up for Koffka's ring illusion now with half the background colored red and half grey. In the top panel the thick contiguous ring appears a homogeneous grey, while on the bottom panel the left hand side of the split ring appears to be cyan. b, As in Fig. 2 a thin contiguous ring appears to be heterogeneously colored, as does a mid-sized ring. Again, as in Fig. 2 upon viewing the rings from bottom to top a ring of the same thickness as in (a) now appears to be heterogeneous. 

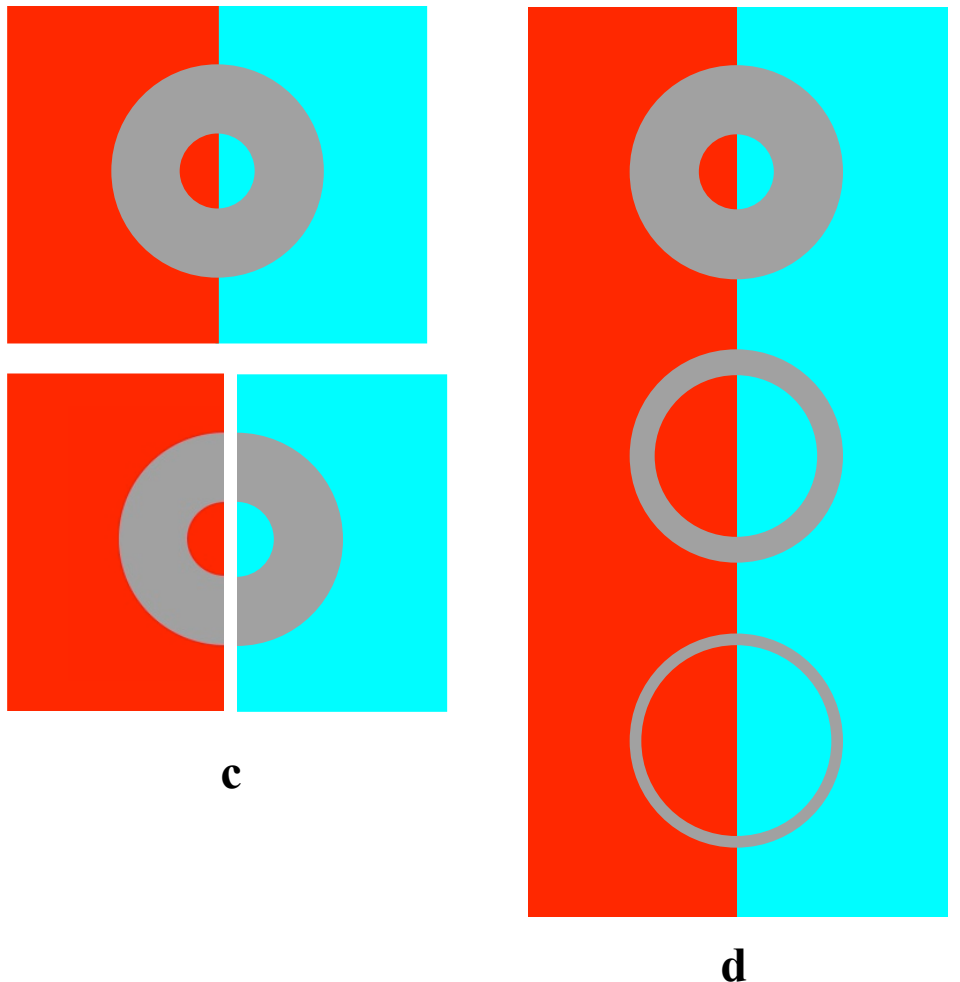

Figure 4 (cont.) c, d A two-colored version of the standard (c) and thin ring and hysteresis (d) effects. 

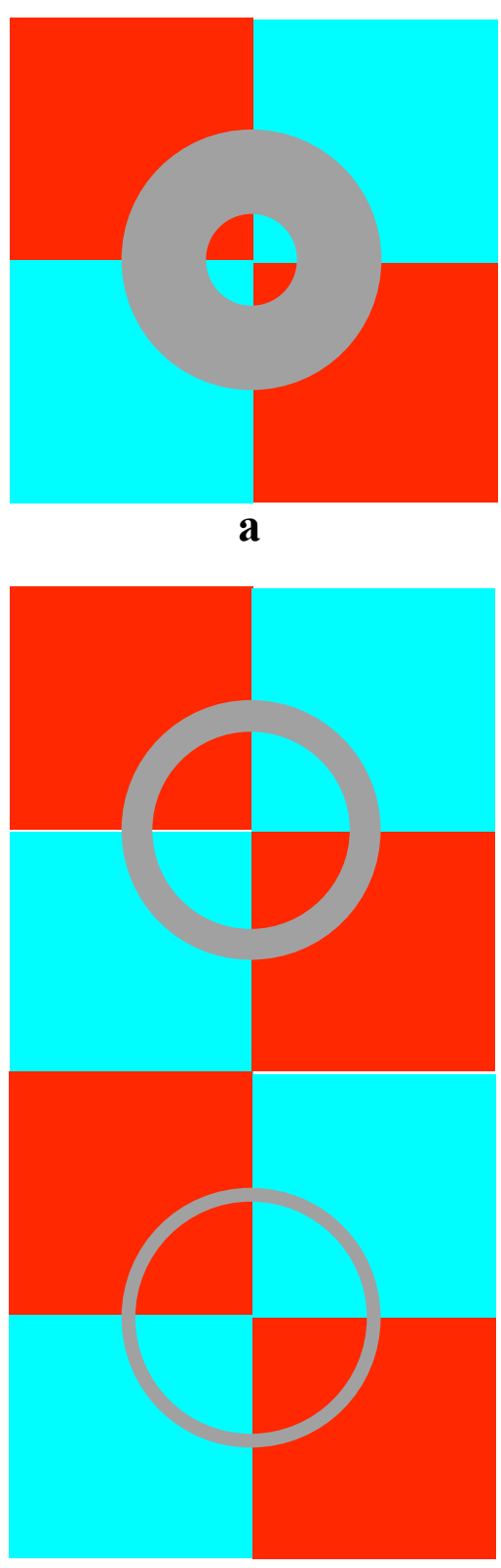

\section{Figure 5 Quadrant version of the ring} effects. a, Koffka's effect for a quadrant background. b, Thin and mid-thickness rings again appear heterogeneous. Also, again, the top the thick ring, (a), appears heterogeneous when viewed after looking first at the thin and mid-sized rings. 


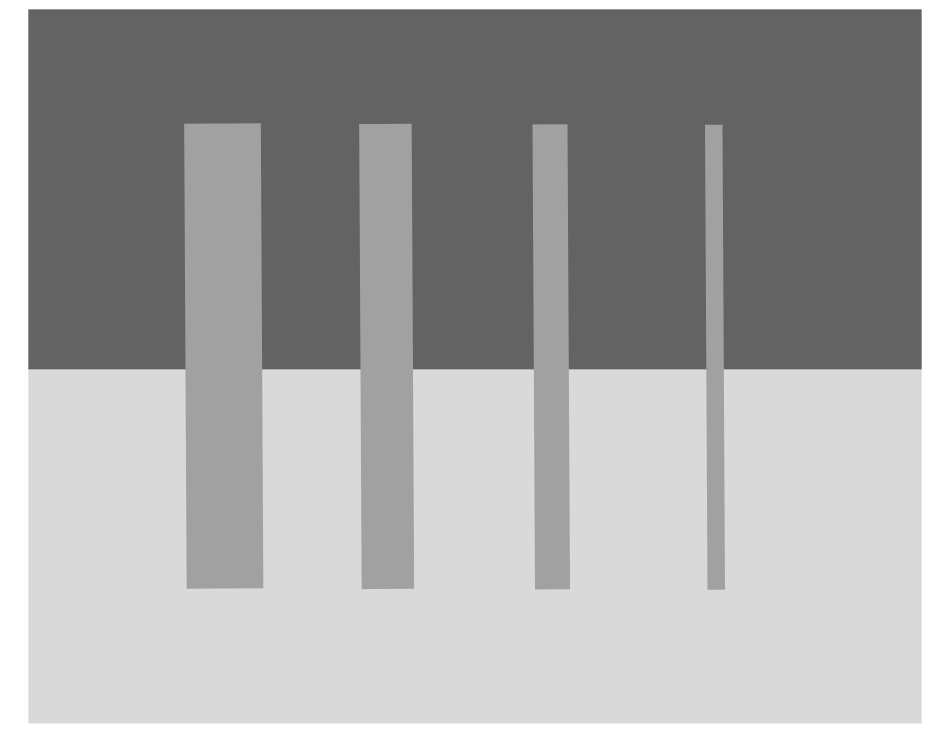

Figure 6

Simultaneous

contrast weakens

with increasing

thickness of the test

patch. a, b Notice

$\mathbf{a}$

that both for

simultaneous

brightness (a), and

color (b) contrast

weaken with

increasing thickness of

the test patch. 


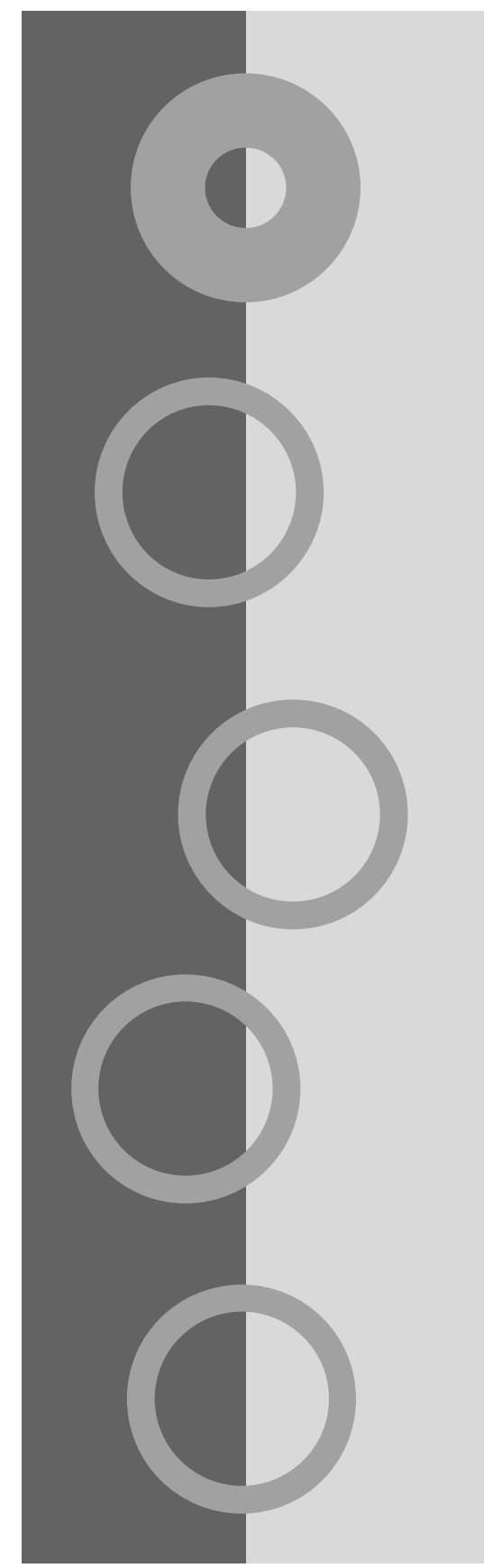

Figure 7 The hysteresis effect is not a simple afterimage. The thin rings appear heterogeneous. The top thick ring appears homogeneous when viewed first, but heterogeneous when viewed after one of the thinner rings. The thick ring appears divided at the border of the two colors of the background, not at the cord location dividing a given thin inducing ring. 